\title{
Somatosensory Electrical Stimulator for Assessment of Current Perception Threshold at Different Frequencies
}

\author{
William Azevedo de Paula1, Renato Zanetti², Laisla Vieira de Almeida ${ }^{1,2}$, Emerson Fachin-Martins ${ }^{3}$, \\ Henrique Resende Martins ${ }^{1}$ \\ ${ }^{1}$ Graduate Program in Electrical Engineering - Universidade Federal de Minas Gerais - Av. Antônio Carlos 6627, 31270- \\ 901, Belo Horizonte, MG, Brazil \\ williamazevedodepaula@hotmail.com; lala.vial@hotmail.com; henriquerm@ufmg.br \\ ${ }^{2}$ CEFET-MG - Centro Federal de Educação Tecnológica de Minas Gerais \\ Av. Amazonas 5253, Belo Horizonte, Brazil \\ zanetti@cefetmg.br \\ ${ }^{3}$ NTAAI - Núcleo de Tecnologia Assistiva, Acessibilidade e Inovação / UnB - Universidade de Brasília \\ Setor A Norte Campus Universitário - Centro Metropolitano, Ceilândia Sul, Brasília - DF, Brazil \\ emersonntaai@gmail.com
}

\begin{abstract}
We aim to introduce the $\mathrm{EE}_{\mathrm{L}} \mathrm{S}$ device, an advantageous somatosensory electrical stimulator composed by hardware, firmware, and software to perform peripheral afferent fibers assessment based on sinusoidal current. We designed the $\mathrm{EE}_{\mathrm{L}} \mathrm{S}$ combining the precision given by STM32 microcontroller and the stability generated by the current source based on a bootstrap topology, a simplified and stable system if compared to a first equipment version. We coded the software as an Android Mobile Application (App) to have compatibility with mobile devices and reduce hardware set up time. Workbench tests shows $\mathrm{EE}_{\mathrm{L}} \mathrm{S}$ system operation capabilities in terms of Total Harmonic Distortion (THD), stimulus linearity, stimuli's frequency spectrum, and maximum current amplitude. The tests' results show an reduction in linearity when compared to the previous device, but the second order coefficient remains 10,000 times less than the first order coefficient. The bootstrap topology allows for a higher stimuli bandwidth up to $10,000 \mathrm{~Hz}$, and the a higher current intensity (11.2 $\mathrm{mA}$ at maximum). Additionally, the App was stable during all tests and considered by us as intuitive and user friendly. Considering all improvements, $\mathrm{EE}_{\mathrm{L}} \mathrm{S}$ could outperform its predecessor, presenting a more intuitive and simple operation to break new grounds on research and clinical applications.
\end{abstract}

Keywords: Somatosensory, Sinusoidal Electrical Stimulator, Sensory Threshold, Current Perception Threshold, Neuropathy.

\section{Introduction}

Electrical stimulation [1], monofilaments [2], and the discrimination between two points [3] are techniques proposed to support the diagnosis of peripheral sensory impairments [2,4]. Among them, those applying electrical stimulation surpass the benefits of mechanical-based techniques by bringing quantitative measures to improve the clinical assessment, such as the Current Perception Threshold (CPT) and Reaction Time (RT). Furthermore, this techniques also can generate neuroselective information to assess different kinds of somatosensorial nerve fibers related to distinct sensations [1].

The selective triggering of peripheral nerve fibers is reached due to the sinusoidal current's properties at different frequencies, not suitably delivered by electrical pulse shape currents [1]. As the first proposed system to assess somatosensorial function by sinusoidal electrical stimulation was the Neurometer ${ }^{\circledR}[4,6]$, presenting the concept of somatosensorial electrical diagnosis in the ' 80 s and followed by the NeuroStim $[5,7,8]$, which added - as its key-innovation - the multifrequency and arbitrary waveform.

In this paper, we introduce the $\mathrm{EE}_{\mathrm{L}} \mathrm{S}$ (acronym from the Portuguese words Estimulador E Eétrico Somatossensorial) aiming at providing a new device to perform sensorial electrical diagnosis by the sinusoidal current, a more robust system composed by hardware, firmware, and software if compared to its predecessor, the NeuroStim. 


\section{Material and Methods}

We followed a modular design when developing $\mathrm{EE}_{\mathrm{L}} \mathrm{S}$, preserving previously tested features with NeuroStim device, and introducing improvements that favor both neuroselectivity and technical performance related to quality and stability of the generated stimuli, as well as usability of the system.

\subsection{Hardware}

We designed the equipment considering two main blocks: Control Unit (UC) and Power Unit (PU), shown in the Fig. 1-A and Fig. 1-B, respectively. The system uses a medical graded voltage source (AC/DC converter, $\pm 12 \mathrm{~V}$ and $45 \mathrm{~W}$ output, complying with EN61000-3-2, class I tests approved, and Class II supported with Class A EMI) to powers the entire system, accomplishing the NBR 60601-1 ABNT regulation for these kinds of equipment, ensuring electrical security to system (Fig. $1)$.

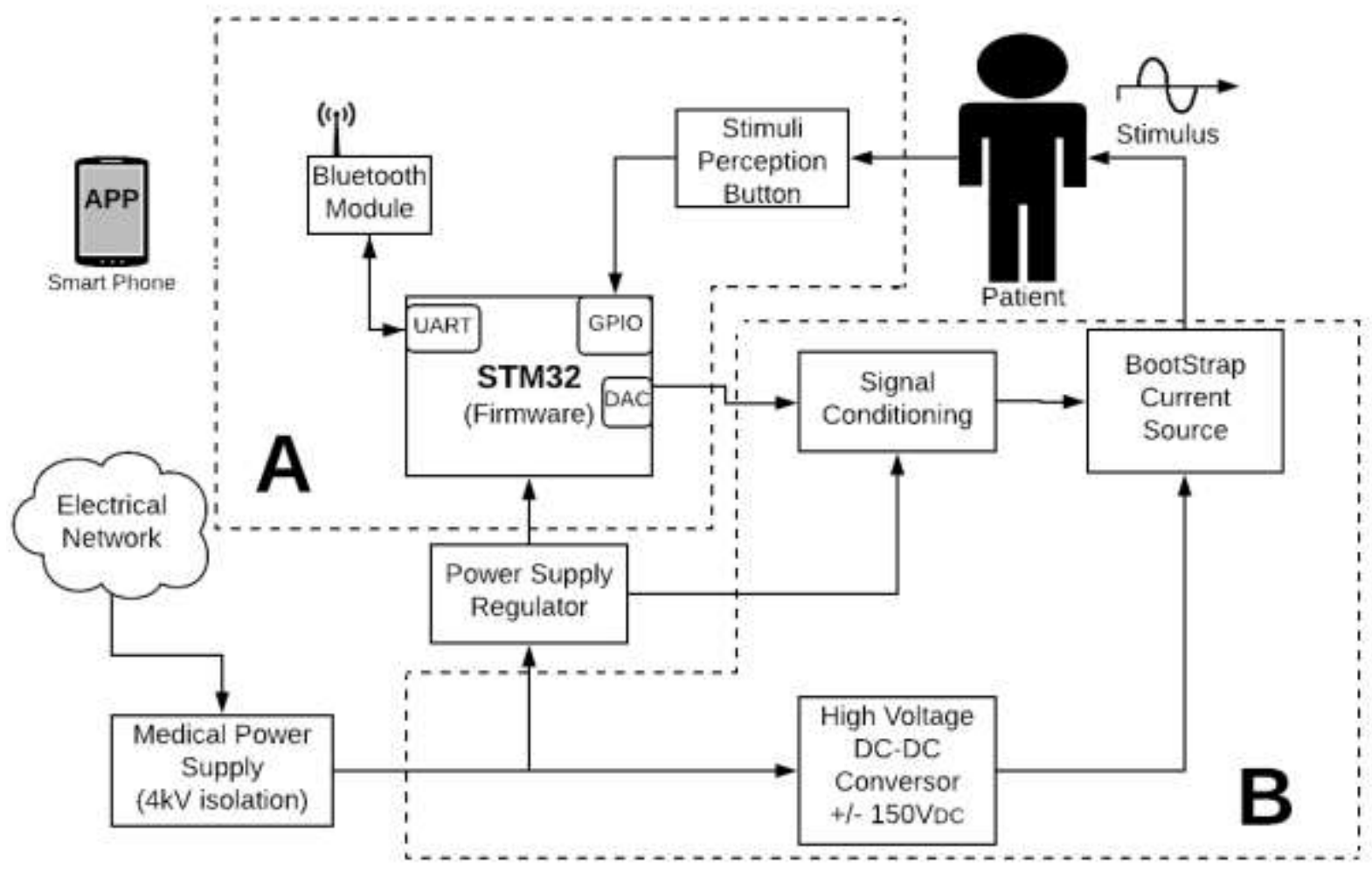

Fig. 1: $\mathrm{EE}_{\mathrm{L}} \mathrm{S}$ hardware block diagram. A - Control Unit. B - Power Unit.

$\mathrm{EE}_{\mathrm{L}} \mathrm{S}$ control unit (Fig. 1-A) is based on STM32F4 microcontroller, which integrates a 32-bit RISC processor, operating with frequency up to $180 \mathrm{MHz}$, providing $512 \mathrm{kB}$ flash memory, $128 \mathrm{kB}$ RAM, and two internal 12-bit Digital to Analog converters (DACs), an essential peripheral as it provides $\mathrm{EE}_{\mathrm{L}} \mathrm{S}$ the capability of generating an sinusoidal output reaching frequencies up to $25 \mathrm{kHz}$ (360 points/ cycle). The signal conditioning circuit transforms the DAC asymmetric output voltage ( 0 to $3.3 \mathrm{~V}$ ) into a symmetric signal (up to $\pm 15 \mathrm{~V}$ ) used as input to the voltage controlled Bootstrap Current Source [9], which can produce outputs with $4.6 \mu \mathrm{A} /$ bit of resolution and 10mA maximum amplitude (also related to the DAC features). The system integrates a Stimulus Perception Button (SPB) to indicates a somatosensory perception. A Bluetooth communication functionality to interface with the proprietary App is provided by an HC-05 Module, reducing setup time and possible problems due to cable connections. 


\subsection{Firmware}

The $\mathrm{EE}_{\mathrm{L}} \mathrm{S}$ firmware controls the hardware by a Finite State Machine (FSM) presented in the Fig. 2. The transitions of the FSM are performed according to commands received from the App to control the hardware operations.

The system can perform stimuli with both sinusoidal and pulse waveform, the latter allows the $\mathrm{EE}_{\mathrm{L}} \mathrm{S}$ to be used for a broader set of applications, for example, eliciting somatosensory evoked potentials by peripheral nerve stimulation. Furthermore, the algorithm enables to control several other parameters of stimulation, such as frequency, amplitude, waveform, number of stimuli, and pulse width when applicable. The firmware set up the Direct Memory Access (DMA) module of the microcontroller to generate the stimulus accessing a DAC peripheral and transferring data from a buffer calculated after receiving stimulus parameter from the App. The DMA ensures a high-speed generation of the analogic output signals that allows $\mathrm{EE}_{\mathrm{L}} \mathrm{S}$ to achieve sinusoidal frequencies up to $25 \mathrm{kHz}$ with a resolution of 360 points per period.

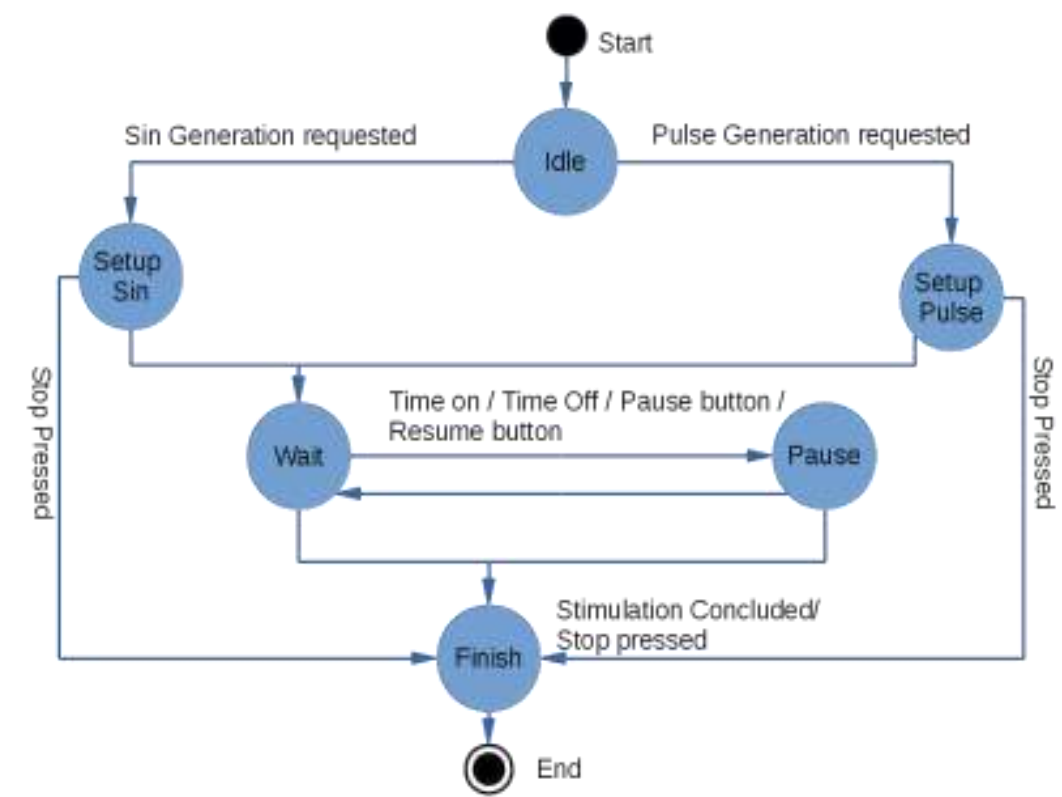

Fig. 2: Representative flowchart of the Finite State Machine (blue circles) and the events (arrows) that cause each state transition.

The communication between firmware and App is based on a master-slave concept, and the former always behaves as a slave responding to commands received from the App. Interrupt Service Routines (ISR) were also conceived for timecritical tasks as, for example, SPB event to measure accurately the patient RT. So, the protocol of stimulation is implemented in the App, which sends a series of state-changing commands to the firmware, controlling the hardware.

The firmware works as a real-time embedded system composed by three blocks. The Command Decoder (name chosen to the first block) works asynchronously to decode Bluetooth data packet and control command priorities - some commands need higher priority due to security precautions. The Central Processor (second block) executes one state machine for each channel, in a parallel way (There is one instance of the FSM per channel). Finally, the last block (Buffer Routine) works as a circular memory in which the Central Processor block searches for commands to execute stimulation protocols.

The FSM performs six different states. On the Idle State, the device is ready for new stimulations, but does not produce any output stimulus on the hardware. The Setup Sin and the Setup Pulse are transitory states, and the FSM configures the DAC, timers and DMA modules that will produce stimulations. Then, it goes automatically to the Wait State. On the Wait State, the FSM awaits for next command meanwhile DMA modules send points to DAC to output the stimulation control signal. On the Pause State, the FSM interrupt the stimulation, turning off the output. The stimulation can be resumed from the point it has stopped. At last, on the Finish State, the FSM indicates the end of the programmed stimulation or that the operator pressed the "stop" button in the App. 


\subsection{Mobile Application - App}

The App works as a Graphical User Interface (GUI), providing full control of $\mathrm{EE}_{\mathrm{L}} \mathrm{S}$ hardware. It was developed as an Android Mobile Application, using AngularJS and Cordova frameworks which allows portability to other platforms.

In the present project, we implemented two different stimulation protocols on the App, Ramp, and Sensory Threshold (ST) Tests, according to described in [8]. This protocol allows the operator to determine the CPT [10] and RT [11] at different frequencies.

\subsection{Tests}

We did execute both operational tests and performance assessment using a $10 \mathrm{k} \Omega$ resistor as the output load. Those tests assessed the bandwidth, the linearity, and Total Harmonic Distortion (THD) of the $\mathrm{EE}_{\mathrm{L}} \mathrm{S}$ output sinusoidal stimulation.

To perform the linearity test we measured output current (IOUT) whereas applying different amplitude values on the App from $0 \mathrm{~mA}$ up to the maximum (at 20 different measuring points) at different frequencies, 250 and 2,000 $\mathrm{Hz}$.

We considered as the maximum amplitude the last value before the system starts producing distortion or saturation at the output stimulus. The linearity grade was obtained using a second-order polynomial regression (using the function "polyfit" of the software Matlab®). The THD was evaluated using the function "thd" of the software Matlab® over the points obtained with a digital oscilloscope at five different frequencies, 1, 5, 250, 2,000, and 3,000 $\mathrm{Hz}$. The results were compared with NeuroStim and the literature.

\section{Results}

The Fig. 3 shows a picture of the entire system. We developed this $E_{L} S$ version with two independents channels to allow different protocols.

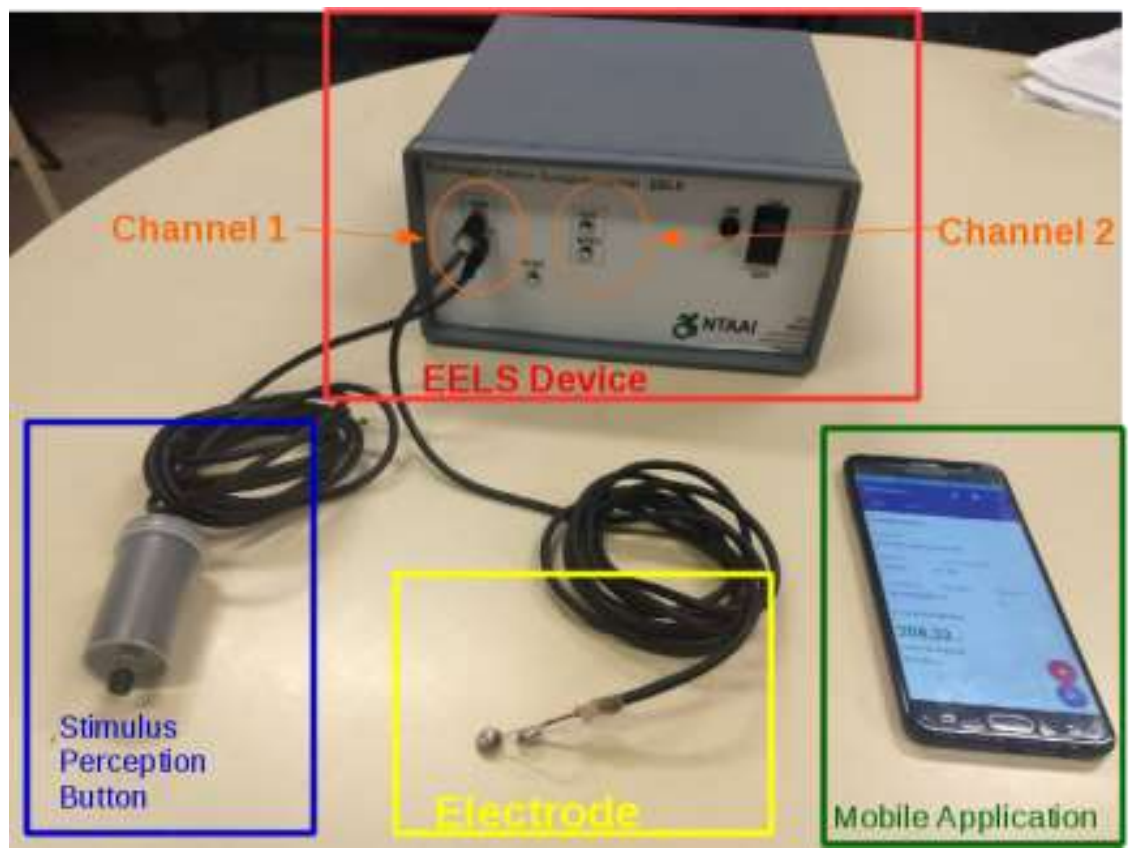

Fig. 3: Overview of the $\mathrm{EE}_{\mathrm{L}} \mathrm{S}$ system components.

Fig. 4 shows a picture of the mounted $\mathrm{EE}_{\mathrm{L}} \mathrm{S}$ printed circuit board for the top and bottom view, highlighting its main blocks, the Control Unit (Microcontroller, Bluetooth module, stimulus perception button connector, and signal conditioning) and Power Unit (High Voltage DC-DC converter, current sources). 

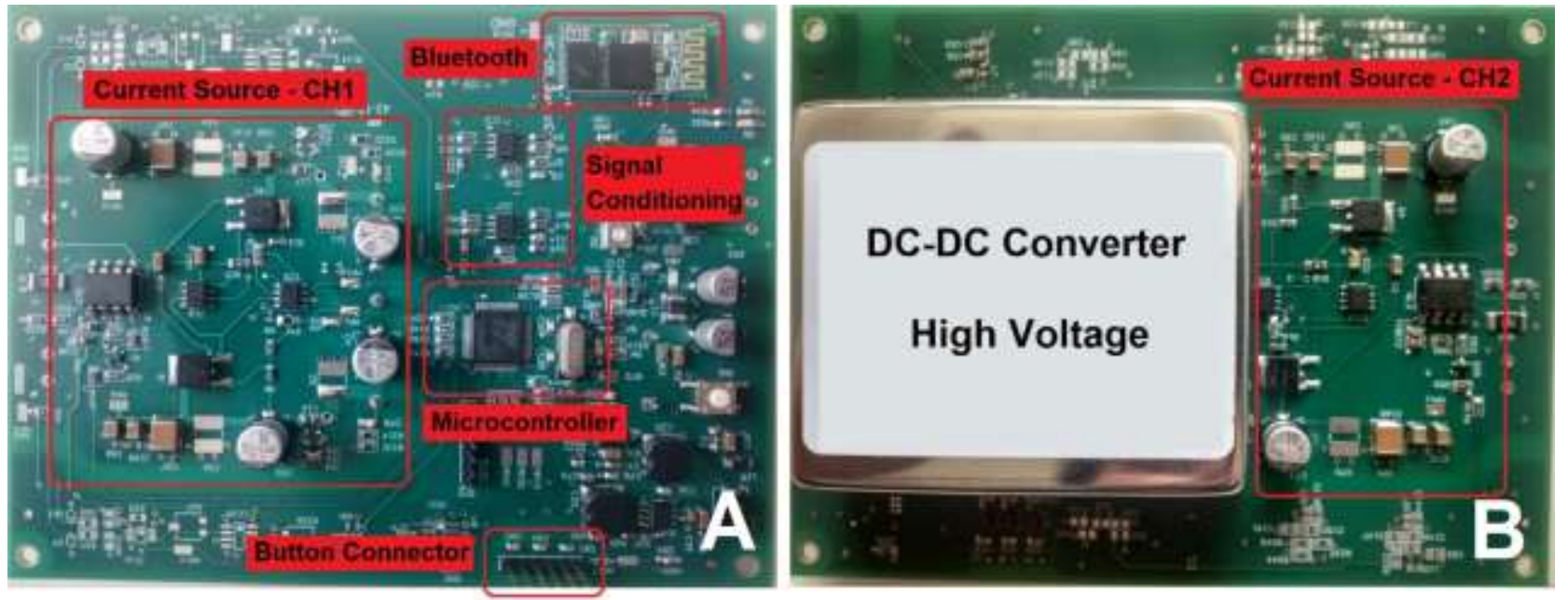

Fig. 4: $E_{\mathrm{L}} \mathrm{S}$ mounted PCB, highlighting its main components for top (A) and bottom (B) sides.

The Fig. 5 shows screens from the App performing different steps of the algorithm, allowing to visualise tabs that represents the two channels $(\mathrm{CH} 1$ and $\mathrm{CH} 2$ ). In each tab, the operator sets up the protocol (Ramp or Sensory Threshold Test) and the parameters of the stimuli (frequency, initial intensity, Time On, Time Off, Increase), controls by mean of the buttons (to start, pause and stop the stimulation), and checks on-line the current intensity and operation status (Paused, Stimulating or Inactive).

When performing the Ramp Test (Fig. 5-A), the operator may choose an initial amplitude and frequency. Following, the operator clicks play button to start the test at the point from which the operation becomes automatic and the EE $E_{L} S$ starts to increase the amplitude until the patient press the SPB. Then, it stops the stimulation and saves the threshold reached in the Ramp Test. The reached threshold becomes green-coloured on the screen. Afterwards, the App offers the ST TEST button opening the next interface (Fig. 5-B).

From this moment, the system begins the ST TEST performing three different steps according to the protocol described in [8]: identification and validation of the CPT and measurement of the RT. The identification proceeds a series of stimuli triggered with intensities vary narrow and around the threshold reached in the Ramp Test to refine the CPT. In the validation, the App delivery randomly eight stimuli (double-blind) to be chosen by the patient. Among all, haf are fake stimuli $(0 \mu \mathrm{A})$ and other half are real stimuli (the refined CPT). Then, the software counting how many fake stimuli were chosen as real stimuli (false positive) and the reverse choice (false negative). The false positive or negative are highlighted on the App by red-coloured X (Fig. 5-B). The validation considers a maximum of two different errors (false positive and negative), do not allowing equal mistakes. Only if undergone the validation, the refined CPT becomes the validated CPT (CPTV). Otherwise, the system displays an "error in diagnosis" message and asks to the operator to repeat or not the ST TEST.

In turn, the measurement of the RT (Fig. 5-C) comprises four stimuli equal to 1.2 times the CPTV (20\% higher than the $\mathrm{CPT}_{\mathrm{V}}$ ) delivered with intercalated by rest times ranging randomly (double-blind) from 1 up to 6 seconds. The mentioned procedure aims to avoid any learning effect. The RT represents the time between the stimulation and the moment at the patient pushes the SPB. How we have four RTs, the App calculates the average of them in milliseconds, as observed on the bottom of the App Screen (Fig. 5-C). All parameters and results are stored in a local database and syncs with a cloud database whenever the mobile device is connected to the internet. 


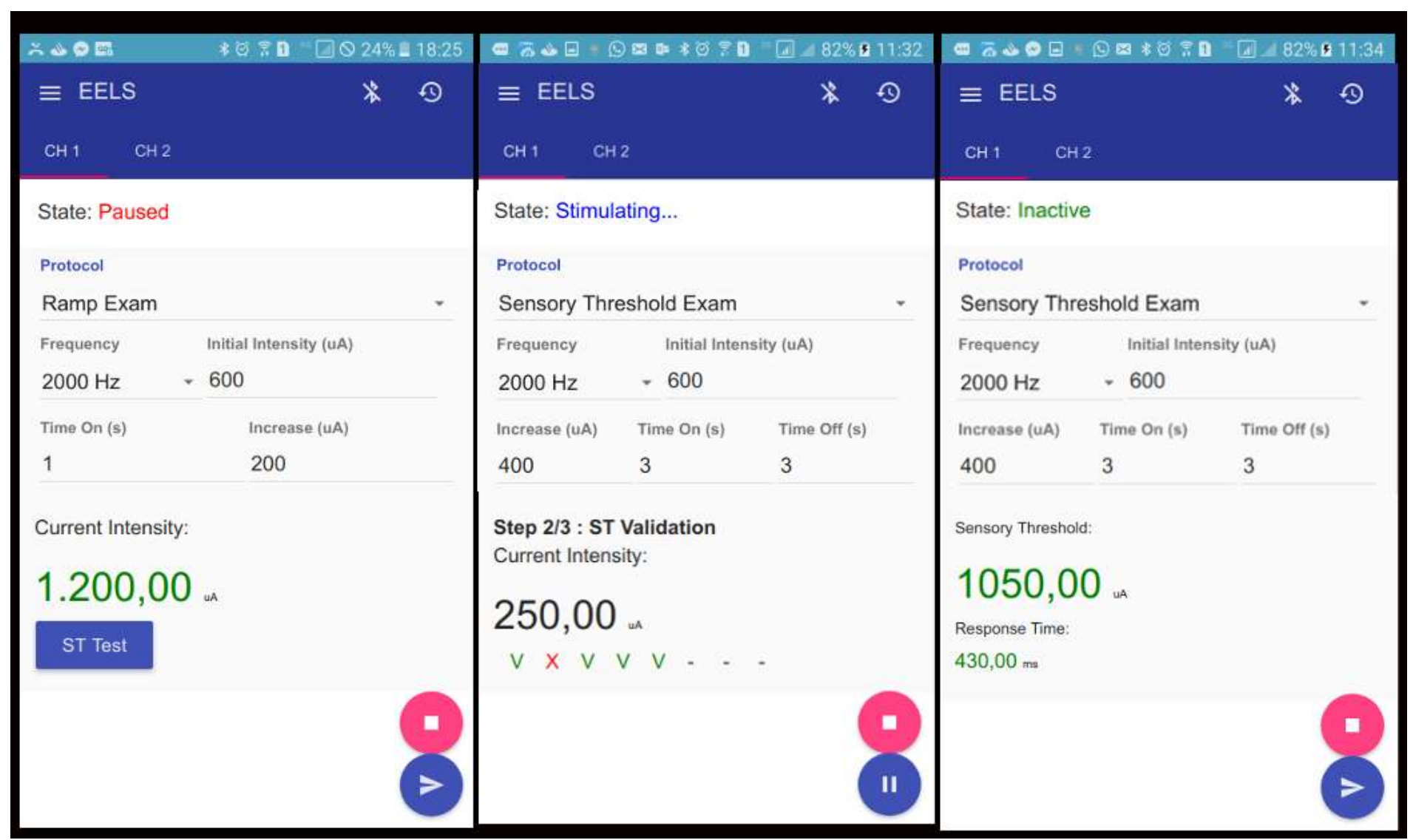

Fig. 5: The $\mathrm{EE}_{\mathrm{L}} \mathrm{S}$ user interface. From left to right: A - the result of the ramp exam; B - the verification step during threshold determination; $\mathrm{C}$ - the obtained threshold and response time.

The system generated sinusoidal current intensity until $11.2 \mathrm{~mA}$ (considering the resistor load of $10 \mathrm{k} \Omega$ ), with $10 \mathrm{kHz}$ bandwidth. Beyond this amplitude, the hardware temperature increases over $60^{\circ} \mathrm{C}$ and distortions were observed in the osciloscope. The Fig. 6 exemplify that our system generated the expected sinusoidal current waveform.

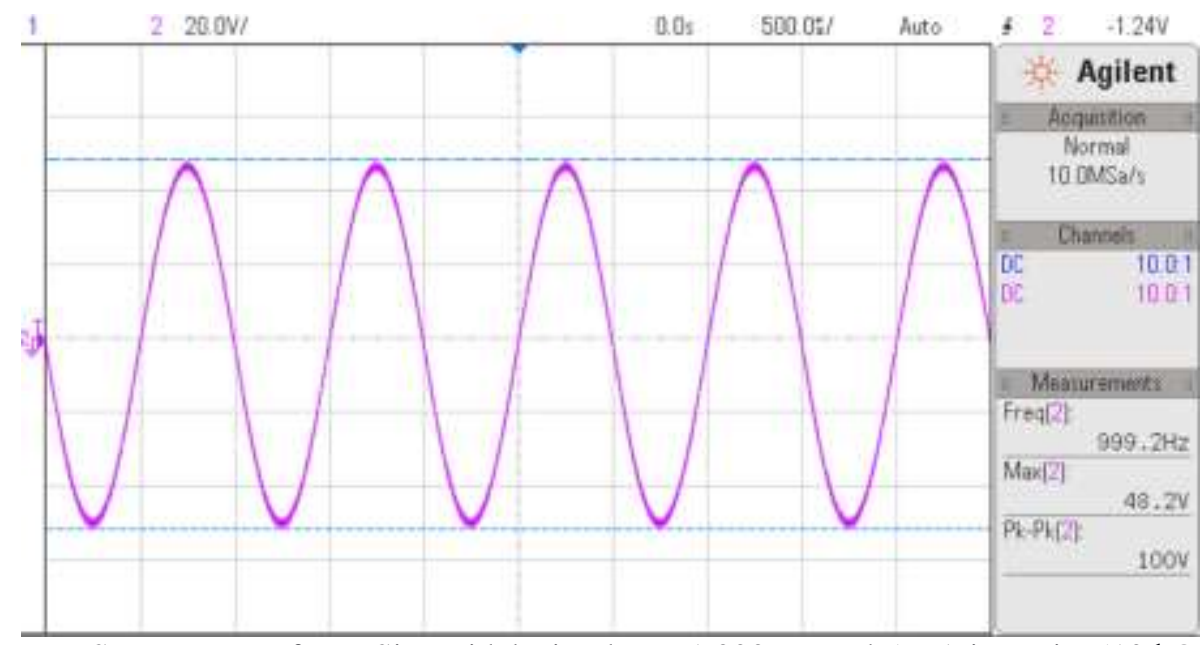

Fig. 6: $\mathrm{EE}_{\mathrm{L}} \mathrm{S}$ output waveform. Sinusoidal stimulus at $1,000 \mathrm{~Hz}$ and $5 \mathrm{~mA}$ intensity ( $10 \mathrm{k} \Omega$ load). 
The equation estimated by the polynomial regration for each pairs of the twenty chosen and measured amplitudes defined the polynomial coefficients presented in the table 1 . We repeated the estimation for $\mathrm{EE}_{\mathrm{L}} \mathrm{S}$ and NeuroStim systems at different frequencies $\left(250\right.$ and $2,000 \mathrm{~Hz}$ ). The $\mathrm{a}_{2} / \mathrm{a}_{1}$ reveals that the $\mathrm{a}_{1}$ was almost 7,000 times greater than the $\mathrm{a}_{2}$ for both frequencies in the $E_{L} S$. For the NeuroStim, the $a_{1}$ was considerably greater, notably at $250 \mathrm{~Hz}$.

By the $\mathrm{a}_{0}$ coefficient analysis, we identified that the $\mathrm{EE}_{\mathrm{L}} \mathrm{S}$ provided values closer than zero in comparison with NeuroStim. Moreover, the NeuroStim increased the $\mathrm{a}_{0}$ coefficient up to values further than zero at 2,000 $\mathrm{Hz}$.

Table 1: $\mathrm{EE}_{\mathrm{L}} \mathrm{S}$ and NeuroStim polynomial coefficients obtained in the linearity test.

\begin{tabular}{|l|c|c|c|c|}
\hline Stimulator/Frequency & $\mid \mathbf{a}_{1|/|} \mathbf{a}_{2 \mid}$ & $\mathbf{a}_{2}$ & $\mathbf{a}_{1}$ & $\mathbf{a}_{\mathbf{0}}$ \\
\hline $\mathrm{EE}_{\mathrm{L}} \mathrm{S} / 250 \mathrm{~Hz}$ & 6,703 & $-1.28 \times 10^{-4}$ & 0.858 & $-1.540 \times 10^{-2}$ \\
\hline $\mathrm{EE}_{\mathrm{L}} \mathrm{S} / 2,000 \mathrm{~Hz}$ & 7,821 & $-1.097 \times 10^{-4}$ & 0.858 & $-2.041 \times 10^{-3}$ \\
\hline NeuroStim / 250 Hz & 241,000 & $-3.15 \times 10^{-5}$ & 7.59 & -0.85 \\
\hline NeuroStim / 2,000 Hz & 83,552 & $-9.12 \times 10^{-5}$ & 7.62 & -7.95 \\
\hline
\end{tabular}

Finally, the waveform distortion analysis disclosed that the NeuroStim produces a THD 7.26 times greater than that one produced by the $\mathrm{EE}_{\mathrm{L}} \mathrm{S}\left(\mathrm{NeuroStim}=1.38 \%\right.$ and $\left.\mathrm{EE}_{\mathrm{L}} \mathrm{S}=0.19 \%\right)$.

\section{Discussions and Conclusions}

Operational tests indicate that $\mathrm{EE}_{\mathrm{L}} \mathrm{S}$ generated sinusoidal current with a high impedance load (10 $\mathrm{k} \Omega$ ). Moreover, hardware, firmware, and App were correctly integrated allowing two stimulation protocols: Ramp and ST Tests. The EE $\mathrm{E}$ hardware reached sinusoidal stimulus up to $10 \mathrm{kHz}$. Literature proposed stimulus with frequencies up to $3 \mathrm{kHz}$, therefore $\mathrm{EE}_{\mathrm{L}} \mathrm{S}$ output bandwidth allows its use for foreseen applications [1,2,4,6,8].

Neither hardware or software problems have occurred during tests. The user interface is easy to use and stable, and the stimulation was conducted as expected. The Bluetooth communication presented enough throughput. In this way, there was not consistent latency when sending commands to the $\mathrm{EE}_{\mathrm{L}} \mathrm{S}$ and there was no connection or packet loss between them.

The $\mathrm{EE}_{\mathrm{L}} \mathrm{S}$ presented considerable performance enhancements over to NeuroStim features, such as, a higher frequency bandwidth ( $0 \mathrm{~Hz}$ to $10 \mathrm{kHz}$ without electrical currents amplitude losses, against $3.19 \mathrm{~Hz}$ to $5,110 \mathrm{~Hz}$ in the NeuroStim), and a smaller THD, that was 7 times less than the predecessor, suggesting less distortion in the $\mathrm{EE}_{\mathrm{L}} \mathrm{S}$ output signal. Moreover, the signal conditioning circuit do not present high-pass filters, so $\mathrm{EE}_{\mathrm{L}} \mathrm{S}$ can compensate output offset dynamically using a designed feedback circuit.

$\mathrm{EE}_{\mathrm{L}} \mathrm{S}$ current source topology is also more stable than NeuroStim, producing higher output power (maximum of 11.2 $\mathrm{mA}$ against $8 \mathrm{~mA}$ for NeuroStim, at $10 \mathrm{k} \Omega$ load). Both systems presented good linearity characteristics (Table 1), as the second order coefficients for polynomial regression present values which can be considered equal zero in compare to the first order coefficients. Furthermore,

Overall, $\mathrm{EE}_{\mathrm{L}} \mathrm{S}$, a new Electrical Stimulator System, has shown considerable improvements if compared to its predecessor and other similar equipment on the literature, achieving good linearity, THD, stimulus power, and resolution that are needed for a quantitative assessment of peripheral neuropathies evolution. Furthermore, $\mathrm{EE}_{\mathrm{L}} \mathrm{S}$ was developed according to security regulation for electromedical equipment, and an experimental setup with volunteers is being proposed for ethics committee approval to launch future research activities.

We concluded, by most of the evaluated features, that the EELS is worthwhile than the previous system, breaking new grounds to clinical and research applications.

\section{Acknowledgments}

The work was supported by CNPq, CAPES, FAPEMIG and FAPDF, Brazil. 


\section{References}

[1] Pimentel et al., "Perceptions and electric sinusoidal current stimulation," Arquivos de Neuro-Psiquiatria, vol. 64, no. 1, pp. 10-13, 2006.

[2] A. Guclu-Gunduz, S. Citaker, B. Nazliel, C. Irkec, "Upper extremity function and its relation with hand sensation and upper extremity strength in patients with multiple sclerosis," NeuroRehabilitation, vol. 30, no. 4, pp. 369-74, 2012.

[3] M. Catley, A. Tabor, B. Wand and G. Moseley, "Assessing tactile acuity in rheumatology and musculoskeletal medicine-how reliable are two-point discrimination tests at the neck, hand, back and foot?", Rheumatology, vol. 52, no. 8, pp. 1454-1461, 2013.

[4] M. Galvão, G. Manzano, N. Braga, et al, "Determination of electric current perception threshold in a sample of normal volunteers," Arquivos de Neuro-Psiquiatria, vol. 64, no. 1, pp. 10-13, 2006.

[5] H. Martins, "Sistema para o estudo do limiar de percepção de corrente elétrica com forma de onda arbitrária," M.S. thesis, Dept. Eng. Eletrica, UFMG, Belo Horizonte, MG, Brazil, 2008.

[6] "Neurometer CPT - Painless Electrodiagnostic Clinical and Laboratory Sensory Nerve Testing Equipment," Neurotron Incorporated Site (2018). [Online]. Available: http://www.neurotron.com/.

[7] H. Martins, "Sistema para avaliação de fibras nervosas periféricas utilizando corrente elétrica senoidal: estudo de caso em hanseníase," Ph.D. dissertation, Dept. Eng. Eletrica, UFMG, Belo Horizonte, MG, Brazil, 2013.

[8] H. Martins, R. Zanetti, C. Santos, G. Manzano and C. Tierra-Criollo, "Current perception threshold and reaction time in the assessment of sensory peripheral nerve fibers through sinusoidal electrical stimulation at different frequencies,"

Revista Brasileira de Engenharia Biomédica, vol. 29, no. 3, pp. 278-285, 2013.

[9] G. King and T. Watkins, "Bootstrapping your op amp yields wide voltage swings," EDN network (1999). [Online]. Available: http://goo.gl/rp90jQ.

[10] "Technology review: The Neurometer® current perception threshold (CPT)," Muscle \& Nerve, vol. 22, no. 4, pp. 523$531,1999$.

[11] E. Félix et al., "Sensations and reaction times evoked by electrical sinusoidal stimulation," Neurophysiologie Clinique/Clinical Neurophysiology, vol. 39, no. 6, pp. 283-290, 2009. 